\title{
ON SIEBENMANN PERIODICITY
}

\author{
Steven Hutt
}

\begin{abstract}
Cappell and Weinberger gave a geometric interpretation of the Siebenmann periodicity phenomena. This near-periodicity on the structure sets of topological manifolds was originally demonstrated in an indirect way from the periodicity of the simply-connected quadratic $L$-groups, see Nicas and Siebenmann (1977). In particular it was shown for a topological manifold $M, \operatorname{dim} M \geq 5$, with structure set $S(M)$, that there is an exact sequence$$
0 \longrightarrow S(M) \longrightarrow S\left(M \times D^{4}, \partial\right) \longrightarrow Z .
$$

Cappell and Weinberger recovered the inclusion in the exact sequence directly by a geometric construction on homotopy equivalences of topological manifolds. More precisely, they lay the foundations for such a construction since the tools employed in Cappell and Weinberger were of the PL-category and so inappropriate for general topological manifolds.
\end{abstract}

\section{Introduction.}

In this paper we give a purely topological account of the Cappell-Weinberger construction. While our construction clearly has its roots in [1] the presentation is somewhat different relying on mapping cylinder neighbourhood technology rather than branched $S^{1}$-fibrations. Furthermore, using the author's theory of Poincaré sheaves [3], the connection between our construction and the algebraic form of Siebenmann periodicity is explicit. The methods appear to be suitable for generalisation to homology manifolds.

The structure set of an $n$-dimensional topological manifold $\mathrm{M}$ is in oneto-one correspondence with the set $S_{n+1}(M)$ of cobordism classes of globally nullcobordant $n$-dimensional quadratic Poincaré sheaves on M. These are sheaves with a quadratic Poincaré-Verdier self-duality [4]. The topological Cappell-Weinberger construction presented in this paper is readily understood in terms of the L-theory of Poincaré sheaves. In particular, there is a corresponding sheaf theoretic exact sequence

$$
0 \longrightarrow S_{n+1}(M) \stackrel{\otimes C P^{2}}{\longrightarrow} S_{n+5}(M) \stackrel{\bar{\partial}_{4}}{\longrightarrow} L_{4}(Z)
$$


where the homomorphism $\otimes \sigma\left(C P^{2}\right)$ is tensoring with the canonical 4-dimensional symmetric algebraic Poincaré complex $\sigma\left(C P^{2}\right)$ associated to $C P^{2}[3]$, and $\bar{\partial}_{4}$ is obtained by Poincare sheaf transversality at some point of $M$. The exact sequence is obtained in a natural way and replaces the difficult calculations of $[\mathbf{1}]$.

\section{The Cappell-Weinberger Map.}

Let $M$ be a compact $n$-dimensional topological manifold and $S(M)$ its structure set. An element of $S(M)$ is an equivalence class of simple homotopy equivalences $h: N \rightarrow M, N$ a topological manifold (where we assume $h$ restricts to a homeomorphism on the boundary). Two such maps $h_{1}: N_{1} \rightarrow M$, $h_{2}: N_{2} \rightarrow M$ are equivalent if there is a homeomorphism $\phi: N_{1} \rightarrow N_{2}$ such that $h_{2} \cdot \phi$ is homotopic (rel $\partial$ ) to $h_{1}$.

In this section we will construct a map between structure sets

$$
\mathrm{CW}: S(M) \longrightarrow S\left(M \times D^{4}, \partial\right) .
$$

Later we will identify this map with Siebenmann's map obtained algebraically by tensoring with the symmetric signature of $C P^{2}$. In what follows, we concentrate on the case $\partial M=\emptyset$ and leave it to the reader to check our claims are valid when $\partial M \neq \emptyset$. (In particular, for $\partial M \neq \emptyset$ the construction yields $h \times 1: \partial N \times D^{4} \rightarrow \partial M \times D^{4}$ on the boundary so that the map to $Z$ is trivial.)

Suppose then a simple homotopy equivalence $h: N \rightarrow M$ of topological manifolds is given, where $\operatorname{dim} N=\operatorname{dim} M \geq 5$. We shall construct a simple homotopy equivalence $h^{\prime}=\mathrm{CW}(h): N^{\prime} \rightarrow M \times D^{4}$ which is a homeomorphism on the boundary. Consider the composition

$$
N \stackrel{h}{\longrightarrow} M \stackrel{i}{\hookrightarrow} M \times D^{3} .
$$

We follow an argument of Pedersen [6] to show that $i \cdot h$ is homotopic to a locally flat embedding. First form the map

$$
\left(N \times D^{3}, N \times S^{2}\right) \stackrel{h \times 1}{\longrightarrow}\left(M \times D^{3}, M \times S^{2}\right)
$$

which is evidently a bundle map over $h$. By Sullivan theory there is a normal obstruction in $[M, G /$ Top $] \cong[N, G /$ Top $]$ to homotoping $h \times 1$ to a homeomorphism. Now Rourke and Sanderson [9] show that (germs of) codimension 3 manifold neighbourhoods of $N$ in which $N$ is locally flatly embedded are in 1-1 correspondence with $\left[N, B \mathrm{Top}_{3}\right]$. The forgetful map $B \mathrm{Top}_{3} \rightarrow B G_{3}$ has fibre $G_{3} / \mathrm{Top}_{3}$ and induces a map

$$
\left[N, B \mathrm{Top}_{3}\right] \longrightarrow\left[N, B G_{3}\right]
$$


taking a manifold neighbourhood $N \subset U$ to the spherical fibration associated to $\partial V \hookrightarrow V \stackrel{p}{\rightarrow} N$, where $(V, \partial V)$ is a mapping cylinder neighbourhood of $N$ in $U$ (which exists by Quinn [7]). But $G_{3} /$ Top $_{3} \cong G /$ Top, [9], so that the normal obstruction to homotoping $h \times 1$ to a homeomorphism (or rather its negative) may be realised by a manifold neighbourhood of $N$ for which $N$ admits a fibre homotopy trivial mapping cylinder neighbourhood in the above sense. Let $(W, \partial W)$ be such a mapping cylinder neighbourhood with retraction $p:(W, \partial W) \rightarrow N$. Let $\tilde{p}:(\tilde{W}, \partial \tilde{W}) \rightarrow N$ denote the associated path space fibration. Then $\tilde{p}$ has fibres $(F, \partial F) \cong\left(D^{3}, S^{2}\right)$ and there is a natural inclusion $j:(W, \partial W) \subset(\tilde{W}, \partial \tilde{W})$ over $N$.

Consider the map $\bar{\psi}$ defined to be the composition

$$
(W, \partial W) \stackrel{j}{\hookrightarrow}(\tilde{W}, \partial \tilde{W}) \stackrel{\tilde{\omega}}{\longrightarrow}\left(N \times D^{3}, N \times S^{2}\right) \stackrel{h \times 1}{\longrightarrow}\left(M \times D^{3}, M \times S^{2}\right)
$$

where $\tilde{\omega}$ is the assumed fibre homotopy trivialisation of $(\tilde{W}, \partial \tilde{W})$. Then by construction the normal obstruction is zero and $\bar{\psi}$ is homotopic, via $H$ say, to a homeomorphism $\psi$. Let $k: N \subset W$ be the inclusion. Then $i \cdot h \simeq \bar{\psi} \cdot k \simeq \psi \cdot k$. In particular $i \cdot h$ is homotopic to a locally flat embedding. Notice that $\bar{\psi}$ is a map over $h$ in the sense that the diagram

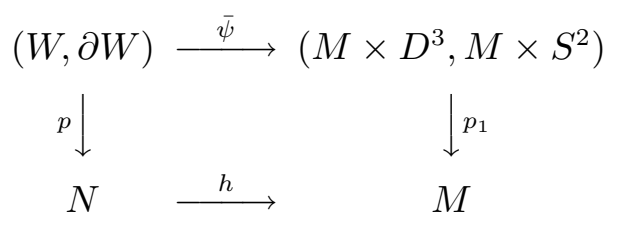

commutes. However $\psi$ will not in general be a map over $h$.

We now construct a simple homotopy equivalence $h^{\prime}: N^{\prime} \rightarrow M \times D^{4}$, which is a homeomorphism on the boundary, and will be the image of $h$ under the Cappell-Weinberger map. Consider the commutative diagram

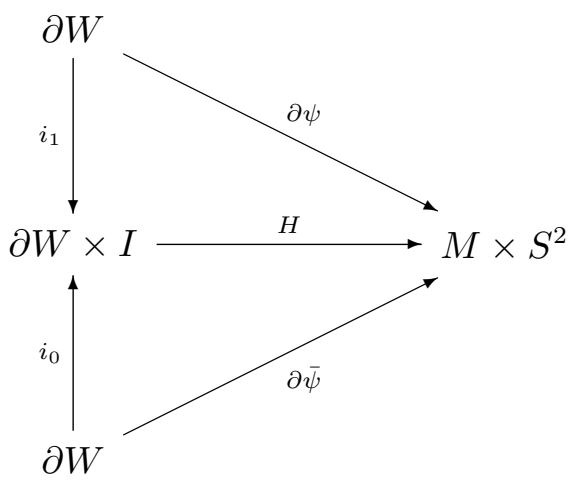

expressing the homotopy $H$ restricted to the boundary. Let $\eta: S^{3} \rightarrow S^{2}$ be the Hopf bundle. Form a new diagram by pulling back the bundle $1 \times \eta$ on 
$M \times S^{2}$

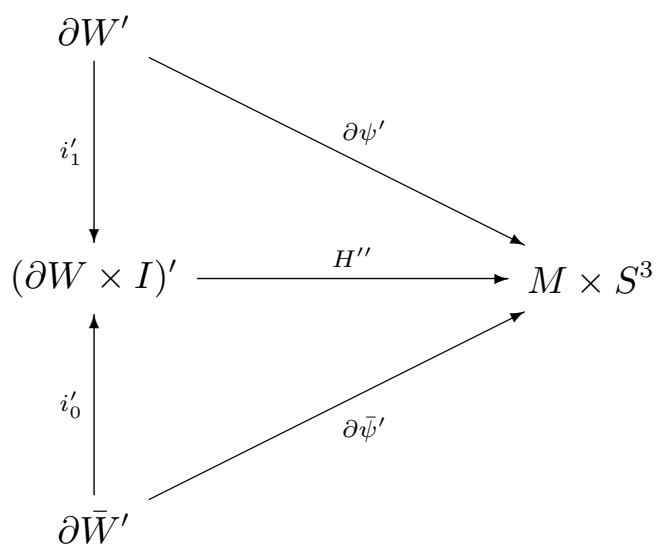

Let $J^{\prime}: \partial W^{\prime} \times I \rightarrow(\partial W \times I)^{\prime}$ be a homotopy over the identity on $\partial W \times I$ starting from the inclusion $\partial W^{\prime} \subset(\partial W \times I)^{\prime}$. Let $g=J^{\prime}(1): \partial W^{\prime} \rightarrow \partial \bar{W}^{\prime} \subset$ $(\partial W \times I)^{\prime}$ and $H^{\prime}=H^{\prime \prime} \cdot J^{\prime}$. Then $H^{\prime}$ is a homotopy $\partial \psi^{\prime} \simeq \partial \bar{\psi}^{\prime} \cdot g$ over $H$. Reparametrising $H^{\prime}$ we obtain a homotopy $H^{\prime}: \partial W^{\prime} \times[1 / 2,1] \rightarrow M \times S^{3}$, where $H_{1 / 2}^{\prime}=\partial \bar{\psi}^{\prime} \cdot g$ and $H_{1}^{\prime}=\partial \psi^{\prime}$.

Write $N^{\prime}$ for the mapping cylinder of the composition $p^{\prime}: \partial W^{\prime} \rightarrow \partial W \stackrel{p}{\rightarrow}$ $N$. Then $N^{\prime}$ is a codimension 4 mapping cylinder neighbourhood of $N$ with boundary $\partial W^{\prime}$. Note that $\partial \psi^{\prime}: \partial W^{\prime} \rightarrow M \times S^{3}$ is a homeomorphism while $\partial \bar{\psi}^{\prime}: \partial \bar{W}^{\prime} \rightarrow M \times S^{3}$ is a map over $h: N \rightarrow M$.

Define

$$
h^{\prime}: N^{\prime}=N \cup_{p^{\prime}} \partial W^{\prime} \times I \longrightarrow M \times D^{4}=M \cup_{p_{1}} M \times S^{3} \times I
$$

on $\partial W^{\prime} \times[1 / 2,1]$ by

$$
\left(H^{\prime}, p_{2}\right): \partial W^{\prime} \times[1 / 2,1] \rightarrow M \times S^{3} \times[1 / 2,1]
$$

and on $N \cup_{p^{\prime}} \partial W^{\prime} \times[0,1 / 2]$ by

$$
h \cup\left(\partial \bar{\psi}^{\prime} \cdot g \times 1\right): N \cup_{p^{\prime}} \partial W^{\prime} \times[0,1 / 2] \rightarrow M \cup_{p_{1}} M \times S^{3} \times[0,1 / 2] .
$$

Lemma 1.1. The class of $h^{\prime}$ in $S\left(M \times D^{4}, \partial\right)$ is independent of the choice of $H: \bar{\psi} \simeq \psi$.

Proof. See appendix.

Definition 1.2. Define the Cappell-Weinberger map

$$
\mathrm{CW}: S(M) \longrightarrow S\left(M \times D^{4}, \partial\right)
$$

by the rule $\mathrm{CW}(h)=h^{\prime}$. 
The topological manifold $N^{\prime}$ may be regarded as a space over $N$ using the obvious projection maps but in general the map $h^{\prime}: N^{\prime} \rightarrow M \times D^{4}$ is not a fibre map over $h$, nor is any representative of $h^{\prime}$ in $S\left(M \times D^{4}, \partial\right)$. Instead we pass to $S\left(M \times C P^{2}\right)$.

Lemma 1.3. There is a canonical map

$$
E: S\left(M \times D^{4}, \partial\right) \longrightarrow S\left(M \times C P^{2}\right)
$$

extending by homeomorphism.

Proof. Let $k: P \rightarrow M \times D^{4}$ be a simple homotopy equivalence of topological manifolds which restricts to a homeomorphism $k_{1}: \partial P \rightarrow M \times S^{3}$ on the boundary. Regard this homeomorphism as an $S^{1}$-equivariant map, with the obvious action on $M \times S^{3}$, and take orbit spaces to obtain a homeomorphism of topological manifolds $k_{2}: \partial P / S^{1} \rightarrow M \times S^{2}$. Define $E(k)$ to be the map

$$
\begin{aligned}
& E(k)=k \cup k_{1} \times 1 \cup k_{2}: P \cup_{\partial P} \partial P \times I \cup \partial P / S^{1} \\
& \longrightarrow M \times D^{4} \cup_{M \times S^{3}} M \times S^{3} \times I \cup M \times S^{3} .
\end{aligned}
$$

Then $E(k)$ extends $k$ by homeomorphism.

Notice that the construction $E$ applied to $h^{\prime}=\mathrm{CW}(h)$ gives the map

$$
E\left(h^{\prime}\right)=h^{\prime} \cup\left(\partial \psi^{\prime} \times 1\right) \cup \partial \psi: \hat{N}=N^{\prime} \cup_{\partial W^{\prime}} \partial W^{\prime} \times I \cup \partial W \longrightarrow M \times C P^{2}
$$

in the above notation. The manifold $\hat{N}$ may be regarded as a space over $N$, again using the obvious projection maps. (As motivation, a result of Edwards [2] shows that the fibres of $\hat{N} \rightarrow N$ may be taken to have the shape of $C P^{2}$. We shall not use this fact directly.) It is readily seen that the homotopies $H, H^{\prime}$ defined above determine a homotopy from $E\left(h^{\prime}\right)$ to the map $\overline{E\left(h^{\prime}\right)}$ given by

$$
\left.E \overline{\left(h^{\prime}\right.}\right)=h \cup\left(\partial \bar{\psi}^{\prime} \cdot g \times 1\right) \cup \partial \bar{\psi}: \hat{N} \longrightarrow M \times C P^{2}
$$

which is a fibre map over $h: N \rightarrow M$. Thus $E\left(h^{\prime}\right) \in S\left(M \times C P^{2}\right)$ is represented by a fibre map.

It is natural to ask whether the diagram

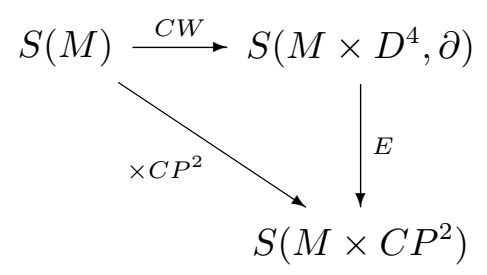


commutes. We shall see later that this is the case, but for now we record

Lemma 1.4. There is a fibre map $\phi: \hat{N} \rightarrow N \times C P^{2}$ over $N$ making the diagram

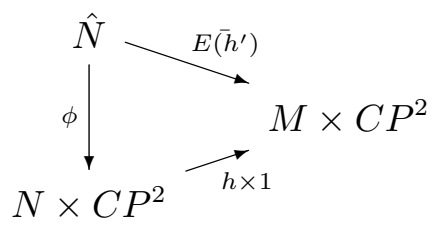

commute.

Proof. Take $\phi: \hat{N} \rightarrow N \times C P^{2}$ to be the map

$$
1 \cup\left(\omega^{\prime} \cdot g \times 1\right) \cup \omega: N \cup \partial W^{\prime} \times I \cup \partial W \longrightarrow N \cup N \times S^{3} \times I \cup N \times S^{2}
$$

where $\omega=\tilde{\omega} \cdot j$ and $\omega^{\prime}=\tilde{\omega}^{\prime} \cdot j^{\prime}$ is its lift.

\section{The Structure Set.}

The structure set of an $n$-dimensional topological manifold $M$ fits into the surgery long exact sequence

$$
\cdots \rightarrow L_{n+1}(\mathbf{Z} \pi) \rightarrow S(M) \rightarrow[M, G / \mathrm{Top}] \rightarrow L_{n}(\mathbf{Z} \pi) \rightarrow \cdots
$$

The quadratic L-groups $L_{n}(\mathbf{Z} \pi)$ are expressed in [8] as the cobordism groups of $n$-dimensional algebraic quadratic Poincaré complexes (AQPCs) of $\mathbf{Z} \pi$ modules. The 1-connective quadratic L-spectrum $\mathbf{L}$. of Ranicki is an $\omega$ spectrum of $\Delta$-sets with simplices $k$-ads of AQPCs (over $\mathbf{Z}$ ). Replacing $G /$ Top by its singular complex, surgery obstructions define an equivalence $G /$ Top $\cong \mathbf{L}$.. In particular by Poincaré duality there is an isomorphism $[M, G /$ Top $] \cong H_{n}(M ; \mathbf{L}$. $)$. Given a triangulation of $M$ the homology group may alternatively be expressed as the cobordism group of stacks of AQPCS on $M,[8]$. As in [8] define the algebraic structure set $S_{*}(M)$ to fit into the algebraic surgery long exact sequence $[8,15.19]$

$$
\cdots \rightarrow L_{n+1}(\mathbf{Z} \pi) \rightarrow S_{n+1}(M) \rightarrow H_{n}(M ; \mathbf{L} .) \stackrel{A}{\rightarrow} L_{n}(\mathbf{Z} \pi) \rightarrow \cdots
$$

where $A$ is the assembly functor taking a stack to its direct limit over the triangulation (after passing to the universal cover). In particular we may regard $S_{n+1}(M)$ as the set of cobordism classes of stacks of $n$-dimensional AQPCs on $M$ with null-cobordant assembly. The long exact sequences (1) 
and (2) are naturally isomorphic and there is defined a 1-1 correspondence $[8,18.5]$

$$
s: S(M) \rightarrow S_{n+1}(M)
$$

Similarly for $i \geq 0$ there are 1-1 correspondences

$$
s: S\left(M \times D^{4 i}, \partial\right) \rightarrow S_{n+4 i+1}(M) .
$$

In [3] the theory of sheaves of AQPCs (i.e. Poincaré sheaves) on an arbitrary locally compact topological space is developed. Poincaré sheaves have good transversality properties so that the cobordism groups of Poincaré sheaves on $M$ form a homology theory $L_{*}(M)$. (In order to recover the 1-connective theory we restrict ourselves to Poincaré sheaves whose 0-component (i.e. apply transversality at a point of $M$ to obtain a 0-dimensional Poincaré sheaf) is trivial.) For example, to a normal (proper) map $(f, b): N \rightarrow M$ of $n$-dimensional topological manifolds is naturally associated an $n$-dimensional Poincaré sheaf $\left(\mathcal{C}_{f}, \psi_{f}\right)$ on $M$ (depending on $\left.b\right)$. Restriction of $(f, b)$ to an open subset of $M$ corresponds to sheaf restriction. Define the sheaf structure set $S_{n+1}^{S h}(M)$ to fit into the long exact sequence

$$
\cdots L_{n+1}(\mathbf{Z} \pi) \rightarrow S_{n+1}^{S h}(M) \rightarrow L_{n}(M) \stackrel{A}{\rightarrow} L_{n}(\mathbf{Z} \pi) \rightarrow \cdots
$$

where $A$ is the assembly functor taking a Poincaré sheaf on $M$ to the AQPC of sections with compact support of the pullback of the sheaf to the universal cover of $M$. For example, the assembly of $\left(\mathcal{C}_{f}, \psi_{f}\right)$ is the surgery obstruction $\sigma(f, b) \in L_{n}(\mathbf{Z} \pi)$. The long exact sequence (3) is isomorphic to (1) and (2) and there is defined a 1-1 correspondence

$$
s: S(M) \rightarrow S_{n+1}^{S h}(M)
$$

Similarly for $i \geq 1$ there are $1-1$ correspondences

$$
s: S\left(M \times D^{4 i}, \partial\right) \rightarrow S_{n+4 i+1}^{S h}(M) .
$$

\section{Siebenmann Periodicity.}

In this section let $S_{*}(M)$ denote the sheaf structure set $S_{*}^{\mathrm{Sh}}(M)$. Let $\left(\mathcal{C}_{C P^{2}}, \phi_{C P^{2}}\right)$ be the canonical 4-dimensional symmetric Poincaré sheaf on $C P^{2}$ with assembly the symmetric Poincaré sheaf $\sigma\left(C P^{2}\right) \in L^{4}(\mathbf{Z})[3,3.1]$. Tensor product defines a map

$$
\otimes \sigma\left(C P^{2}\right): S_{*}(M) \rightarrow S_{*+4}(M),
$$


fitting into the commutative diagram

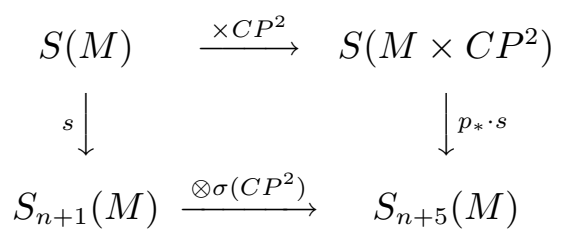

where $p_{*}: S_{n+5}\left(M \times C P^{2}\right) \rightarrow S_{n+5}(M)$ is induced by the projection map. The following theorem identifies the Cappell-Weinberger map algebraically with $\otimes \sigma\left(C P^{2}\right)$.

Theorem 3.1. The diagram

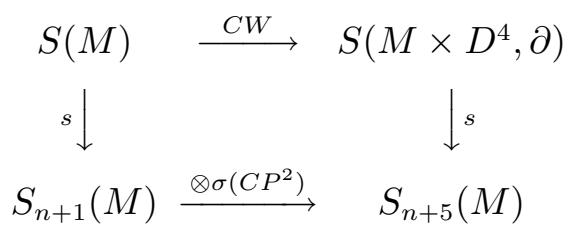

commutes.

Proof. We shall show that the diagram

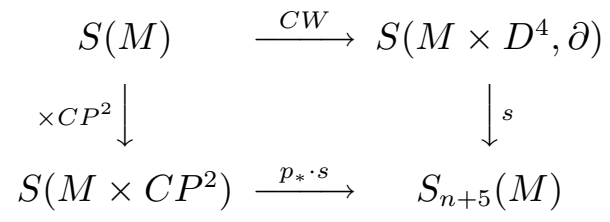

commutes. The theorem will follow from the commutativity of diagram (1). Since $E$ is extension by homeomorphism the diagram

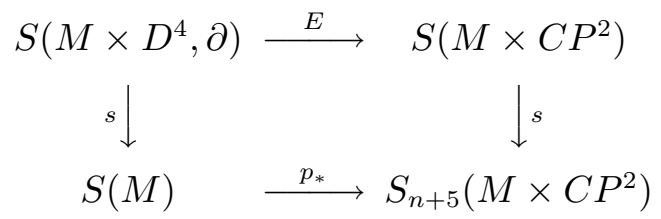

commutes. Suppose then $h \in S(M)$ is given and $h^{\prime}=C W(h) \in S(M \times$ $\left.D^{4}, \partial\right)$. As above $\left.E\left(h^{\prime}\right)=E \overline{\left(h^{\prime}\right.}\right) \in S\left(M \times C P^{2}\right)$ so that in $S_{n+5}(M)$ we have

$$
\left.s\left(h^{\prime}\right)=p_{*}\left(s\left(E\left(h^{\prime}\right)\right)\right)=p_{*}\left(s\left(E \overline{\left(h^{\prime}\right.}\right)\right)\right) .
$$

Recall the map $\phi: \hat{N} \rightarrow N \times C P^{2}$ over $N$ satisfying $(h \times 1) \cdot \phi=\bar{E}\left(h^{\prime}\right)$. Then $\phi$ induces a map of $(n+4)$-dimensional Poincaré sheaves on $M \times C P^{2}$

$$
\left.\phi_{*}: s(h \times 1) \rightarrow s\left(E \overline{\left(h^{\prime}\right.}\right)\right) .
$$


Assume for the moment that the projection of this map to $M$ is a quasiisomorphism of $(n+4)$-dimensional Poincaré sheaves on $M$

$$
\left.p_{*}\left(\phi_{*}\right): p_{*}(s(h \times 1)) \stackrel{\simeq}{\rightarrow} p_{*}\left(s\left(E \overline{\left(h^{\prime}\right.}\right)\right)\right) .
$$

The algebraic mapping cylinder of a quasi-isomorphism of Poincaré sheaves determines a Poincaré cobordism between the ends so that in $S_{n+5}(M)$ we have

$$
\left.p_{*}\left(s\left(E \overline{\left(h^{\prime}\right.}\right)\right)\right)=p_{*}(s(h \times 1)) .
$$

Together with (4) we have

$$
S(C W(h))=s\left(h^{\prime}\right)=p_{*}(s(h \times 1))
$$

so that diagram (2) commutes.

It remains to show that (5) is a quasi-isomorphism. For a space $X$ let $\mathcal{C}_{X}$ be the Godement canonical resolution of the constant sheaf $\mathbf{Z}_{X}$ on $X[3 ; 2]$. The map $\phi: \hat{N} \rightarrow N \times C P^{2}$ over $N$ determines a map of sheaves on $N$

$$
h: p_{*} \mathcal{C}_{N \times C P^{2}} \rightarrow \hat{p}_{*} \mathcal{C}_{\hat{N}} .
$$

It is immediate from the construction of the functor $s: S\left(M \times C P^{2}\right) \rightarrow$ $S_{n+5}\left(M \times C P^{2}\right)$ that we need only show (6) is a quasi-isomorphism. Recall then the construction of $\phi$. It is the composition of maps

$$
\phi_{1}=1 \cup(g \times 1) \cup 1: N \cup \partial W^{\prime} \times I \cup \partial W \longrightarrow N \cup \partial \bar{W}^{\prime} \times I \cup \partial W
$$

and

$$
\phi_{2}=1 \cup\left(\omega^{\prime} \times 1\right) \cup \omega: N \cup \partial \bar{W}^{\prime} \times I \cup \partial W \longrightarrow N \cup N \times S^{3} \times I \cup N \times S^{2} .
$$

Now by construction $g: \partial W^{\prime} \rightarrow \partial \bar{W}^{\prime}$ is a fibre homotopy equivalence over $\partial W$ so that $p_{*}\left(\phi_{1 *}\right)$ is a quasi-isomorphism, $[4,2.7 .7]$. The map $\omega$ is the composition

$$
\partial W \stackrel{j}{\hookrightarrow} \partial \tilde{W} \stackrel{\tilde{\omega}}{\longrightarrow} N \times S^{2}
$$

where $\tilde{\omega}$ is a fibre homotopy equivalence over $N$ and $j$ is the natural inclusion of $\partial W$ (over $N$ ) into the associated path space fibration. But $\partial W$ is the boundary of a mapping cylinder neighbourhood of the topological manifold $N$ so that by $\left[\mathbf{3}\right.$, Theorem 3.8 (proof)] the map $j_{*}: \tilde{p}_{*} \mathcal{C}_{\tilde{\partial} W} \rightarrow p_{*} \mathcal{C}_{\partial W}$ is a quasi-isomorphism. The result follows.

Corollary 3.2. The sequence

$$
0 \rightarrow S(M) \stackrel{C W}{\rightarrow} S\left(M \times D^{4}, \partial\right) \stackrel{\partial_{4}}{\rightarrow} L_{4}(\mathbf{Z})
$$

is exact, where $\partial_{4}$ takes a homotopy equivalence $P \rightarrow M \times D^{4}$ to the splitting invariant of $D^{4} \subset M \times D^{4}$. 
Proof. There is a commutative diagram

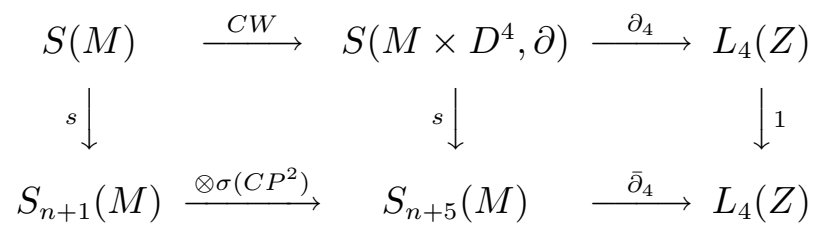

with vertical maps 1-1 correspondences. The map $\bar{\partial}_{4}$ is obtained by making a Poincaré sheaf transverse to a point of $M$ and taking the associated 4dimensional Poincaré sheaf over that point. Thus it is sufficient to show that

$$
0 \rightarrow S_{n+1}(M) \stackrel{\otimes \sigma\left(C P^{2}\right)}{\longrightarrow} S_{n+5}(M) \stackrel{\bar{\partial}}{\rightarrow} L_{4}(\mathbf{Z})
$$

is exact. Let $\bar{S}_{n+1}(M)$ be the 4-periodic sheaf structure set on $M$ obtained by relaxing the 1-connective condition. Then the map $\otimes \sigma\left(C P^{2}\right): \bar{S}_{n+1}(M) \rightarrow$ $S_{n+5}(M)$ is an isomorphism under which the sequence (7) corresponds to the exact sequence

$$
0 \rightarrow S_{n+1}(M) \stackrel{i}{\rightarrow} \bar{S}_{n+1}(M) \stackrel{\bar{\partial}_{0}}{\rightarrow} L_{0}(\mathbf{Z})
$$

where $i$ is the natural map and $\bar{\partial}_{0}$ takes a Poincaré sheaf to its 0 -component. This proves the corollary.

\section{Appendix.}

Proof of Lemma 1.1 Suppose we have constructed the map $\bar{\psi}$ as above. Let $H_{1}$ and $H_{2}$ be two choices of homotopies from $\bar{\psi}$ to homeomorphisms $\psi_{1}$ and $\psi_{2}$ respectively. Let $-H_{2}+H_{1}$ be the obvious homotopy from $\psi_{2}$ to $\psi_{1}$. By a relative version of the embedding argument above there is a homotopy $K$ $($ rel $W \times\{0,1\})$ from $-H_{2}+H_{1}$ to an isotopy $\mathcal{H}$ from $\psi_{2}$ to $\psi_{1}$. Restricting to $\partial W$ gives a map

$$
\partial K: \partial W \times I \times I \rightarrow M \times S^{2}
$$

such that $\partial K_{\mid \partial W \times I \times\{0\}}=-H_{2}+H_{1}$ and $\partial K_{\mid \partial W \times I \times\{1\}}=\partial \mathcal{H}$ is an isotopy from $\partial \psi_{2}$ to $\partial \psi_{1}$. As before let $\partial K^{\prime}:(\partial W \times I \times I)^{\prime} \rightarrow M \times S^{3}$ be the pullback of the bundle $1 \times \eta$ across the map $\partial K$ and $J^{\prime}: \partial W_{1}^{\prime} \times I \times I \rightarrow(\partial W \times I \times I)^{\prime}$ a homotopy over the identity on $\partial W \times I \times I$ starting from the inclusion $\partial W_{1}^{\prime} \times\{1\} \times I \subset(\partial W \times I \times I)^{\prime}$. Let

$$
g_{1}=J_{\mid \partial W_{1}^{\prime} \times\{1 / 2\} \times\{0\}}^{\prime}: \partial W_{1}^{\prime} \rightarrow \partial \bar{W}^{\prime} \subset(\partial W \times I \times I)^{\prime}
$$


and

$$
g=J_{\mid \partial W_{1}^{\prime} \times\{0\} \times\{0\}}^{\prime}: \partial W_{1}^{\prime} \rightarrow \partial W_{2}^{\prime} \subset(\partial W \times I \times I)^{\prime}
$$

and

$$
\bar{g}=J_{\mid \partial W_{1}^{\prime} \times\{0\} \times\{1\}}^{\prime}: \partial W_{1}^{\prime} \rightarrow \partial W_{2}^{\prime} \subset(\partial W \times I \times I)^{\prime}
$$

so that $\bar{g}$ is a homeomorphism over the identity on $\partial W$. Let

$$
H_{1}^{\prime \prime}=J_{\mid \partial W_{1}^{\prime} \times[1 / 2,1] \times\{0\}}^{\prime}
$$

and

$$
H^{\prime \prime}=J_{\mid \partial W_{1}^{\prime} \times[0,1] \times\{0\}}^{\prime}
$$

and

$$
\mathcal{H}^{\prime \prime}=J_{\mid \partial W_{1}^{\prime} \times[0,1] \times\{1\}}^{\prime}
$$

Then $H_{1}^{\prime}=\partial K \cdot H_{1}^{\prime \prime}$ is a homotopy $\partial \bar{\psi}^{\prime} \simeq \partial \psi_{1}^{\prime}, H^{\prime}=\partial K^{\prime} \cdot H^{\prime \prime}$ is a homotopy $\partial \psi_{2}^{\prime} \cdot g \simeq \partial \psi_{1}^{\prime}$ and $\mathcal{H}^{\prime}=\partial K^{\prime} \cdot \mathcal{H}^{\prime \prime}$ is an isotopy $\partial \psi_{2}^{\prime} \cdot \bar{g} \simeq \partial \psi_{1}^{\prime}$. Finally let $H_{2}^{\prime \prime}$ be a homotopy over the identity on $\partial W \times[0,1 / 2] \times\{0\}$ starting at the inclusion $\partial W_{2}^{\prime} \subset(\partial W \times I \times I)^{\prime}$. Set $g_{2}=\left.H_{2}^{\prime \prime}\right|_{2} ^{\prime} \times\{0\}: \partial W_{2}^{\prime} \rightarrow \partial \bar{W}^{\prime}$. Then $H_{2}^{\prime}=\partial K^{\prime} \cdot H_{2}^{\prime \prime}$ is a homotopy $\partial \psi_{2}^{\prime} \simeq \partial \bar{\psi}^{\prime} \cdot g_{2}$.

Define a homeomorphism $\phi: N_{1}^{\prime} \rightarrow N_{2}^{\prime}$. On $N \cup_{p_{1}^{\prime}} \partial W_{1}^{\prime} \times[0,1 / 2]$ set $\phi$ to be the map

$$
1 \cup(\bar{g} \times 1): N \cup_{p_{1}^{\prime}} \partial W_{1}^{\prime} \times[0,1 / 2] \rightarrow N \cup_{p_{2}^{\prime}} \partial W_{2}^{\prime} \times[0,1 / 2]
$$

and on $\partial W_{1}^{\prime} \times[1 / 2,1]$ to be

$$
\left(\left(\partial \psi_{2}^{\prime}\right)^{-1} \cdot \mathcal{H}^{\prime}, p_{2}\right): \partial W_{1}^{\prime} \times[1 / 2] \rightarrow \partial W_{2}^{\prime} \times[1 / 2,1] .
$$

Notice that on the boundary $\partial W_{1}^{\prime}$ of $N_{1}^{\prime}$ we have $\left(h_{2}^{\prime} \cdot \phi\right)_{\mid \partial W_{1}^{\prime}}=h_{1 \mid \partial W_{1}^{\prime}}^{\prime}$. We claim $h_{2}^{\prime} \cdot \phi \simeq h_{1}^{\prime}\left(\right.$ rel $\left.\partial W_{1}^{\prime}\right)$. First note there exists a homotopy $G$ from $\partial \bar{\psi}^{\prime} \cdot g_{1}$ to $\partial \bar{\psi}^{\prime} \cdot g_{2} \cdot g$ over the identity on $\partial W$ and such that $H_{1}^{\prime}+G$ is homotopic (rel end points) to $H^{\prime}+H_{2}^{\prime} \cdot g$. This follows from elementary lifting properties. Using the homotopy $G$ we obtain a homotopy $\left(\right.$ rel $\left.\partial W_{1}^{\prime}\right)$ from $h_{1}^{\prime}$ to a map $h^{\prime \prime}: N_{1}^{\prime} \rightarrow M \times D^{4}$ defined on $N \cup_{p_{1}^{\prime}} \partial W_{1}^{\prime} \times[0,1 / 2]$ to be

$$
1 \cup\left(\partial \bar{\psi}^{\prime} \cdot g_{2} \cdot g \times 1\right): N \cup_{p_{1}^{\prime}} \partial W_{1}^{\prime} \times[0,1 / 2] \rightarrow M \cup_{p_{1}} M \times S^{3} \times[0,1 / 2]
$$


and on $\partial W_{1}^{\prime} \times[1 / 2,3 / 4]$ to be

$$
\left(H_{2}^{\prime} \cdot g, p_{2}\right): \partial W_{1}^{\prime} \times[1 / 2,3 / 4] \rightarrow M \times S^{3} \times[1 / 2,3 / 4]
$$

and on $\partial W_{1}^{\prime} \times[3 / 4,1]$ to be

$$
\left(H^{\prime}, p_{2}\right): \partial W_{1}^{\prime} \times[3 / 4,1] \rightarrow M \times S^{3} \times[3 / 4,1] .
$$

Using the map $J^{\prime}$ homotope $H_{2}^{\prime}$ and $H^{\prime}$ over $\partial W \times I \times\{0\}$ to maps over $\partial W \times I \times\{1\}$. This determines a homotopy (rel $\partial W_{1}^{\prime}$ ) from $h_{1}^{\prime \prime}$ to a map $h_{1}^{\prime \prime \prime}: N_{1}^{\prime} \rightarrow M \times D^{4}$ defined on $N \cup_{p_{1}^{\prime}} \partial W_{1}^{\prime} \times[0,1 / 2]$ to be

$$
1 \cup\left(\partial \psi_{2}^{\prime} \cdot g_{2} \cdot \bar{g} \times 1\right): N \cup_{p_{1}^{\prime}} \partial W_{1}^{\prime} \times[0,1 / 2] \rightarrow M \cup_{p_{1}} M \times S^{3} \times[0,1 / 2]
$$

and on $\partial W_{1}^{\prime} \times[1 / 2,3 / 4]$ to be

$$
\left(H_{2}^{\prime} \cdot \bar{g}, p_{2}\right): \partial W_{1}^{\prime} \times[1 / 2,3 / 4] \rightarrow M \times S^{3} \times[1 / 2,3 / 4]
$$

and on $\partial W_{1}^{\prime} \times[3 / 4,1]$ to be

$$
\left(\mathcal{H}^{\prime}, p_{2}\right): \partial W_{1}^{\prime} \times[3 / 4,1] \rightarrow M \times S^{3} \times[3 / 4,1] .
$$

Clearly $h_{1}^{\prime \prime \prime} \simeq h_{2}^{\prime} \cdot \phi\left(\right.$ rel $\left.\partial W_{1}^{\prime}\right)$.

\section{References}

[1] S. Cappell and S. Weinberger, A geometric interpretation of Siebenmann's periodicity phenomenon, Proceedings, 1985, Georgia Conference on Geometry and Topology, Decker, (1987), 47-52.

[2] R.D. Edwards, Topological regular neighbourhoods, unpublished.

[3] S. Hutt, Poincaré sheaves on topological spaces, preprint.

[4] M. Kashiwara and P. Schapira, Sheaves on manifolds, Grundlehren der Math. Wissenschaften, 292, Springer-Verlag, 1990.

[5] A. Nicas, Induction theorems for groups of homotopy manifold structure sets, Memoirs AMS, 267 (1982).

[6] E.K. Pedersen, Embeddings of topological manifolds, Michigan Journal of Mathematics, (1975), 440-447.

[7] F. Quinn, Ends of maps I, Ann. Math., 110 (1979), 275-331.

[8] A.A. Ranicki, Algebraic L-theory and topological manifolds, Cambridge Tracts in Mathematics, 102, Cambridge University Press, 1992.

[9] C.P. Rourke and B.J. Sanderson, On topological neighbourhoods, Comp. Math., 22 (1970), 387-424.

[10] L. Siebenmann, Approximating cellular maps with homeomorphisms, Topology, 11 (1972), 271-294. 
[11] L. Siebenmann, Periodicity in topological surgery, Foundational Essays on Topological Manifolds by R. Kirby and L. Siebenmann, Princeton University Press, 1977.

Received March 13, 1996 and revised February 20, 1997.

permanent address:

E-mail address: hutt@math.uchicago.edu

Seoul National University

SEOUL 151-742, KorEA

E-mail address: hutt@math.snu.ac.kr 